\title{
Behavioral Analysis of Sustained Individual Investors
}

\author{
Antti Paatela \\ University of Lausanne \\ Jordi Weiss \\ University of Lausanne
}

\section{Abstract}

With a direct access to 32 self-directed investors from two European countries and the USA, we found that with experience, investors learn to mitigate well-studied behavioral biases while new biases and convictions emerge. The explorative interview method revealed reasons for seemingly irrational behavior not discovered by existing empirical studies using aggregated quantitative data. Thematic analyses were done using open coding and predefined concepts of mainstream and behavioral finance. The findings were contrasted with empirical literature and validated with expert interviews. Learning from mistakes, investors in our sample acknowledged the presence of emotions and built ways to mitigate behavioral issues. We found that overconfidence referenced in numerous studies diminishes after initial enthusiasm; underconfidence may emerge after painful losses. Illusion of control could not be identified. Instead, investors reported feeling of insufficient control on their investments. An important new bias candidate, tangibility bias was discovered which makes investors accept lower financial utility if they feel being in control of their investments. Tangibility bias contributes to less efficient portfolios due to the priority for small number of well-known investments instead of well-diversified but not transparent funds. Beyond decision-making biases, investors had developed experiencebased convictions which may be rational or unfounded. Taking into account non-financial motivations, we argue that seemingly irrational actions have a purpose and efficacy.

Keywords: self-directed investing, behavioral biases, decision-making, ecological rationality, portfolio management, underconfidence

\section{Introduction}

Existing theories in the Finance field such as Utility maximization, Efficient markets, Modern portfolio theory or Risk/return interdependence expose general principles of what a rational agent should do and avoid doing when investing money. These 
principles hardly translate into actionable methods, in part because the models are based on strong hypotheses - both about individuals and about the market - that are not satisfied in real life.

Professional investors may use frameworks grounded in these theories, but individual investors are known to largely move away for them, and research on individual investors "as a group" has shown that deviation from such guidelines leads to underperformance compared to the market.

Pooling individual investors creates a risk of masking important individual elements, as these people willingly decided to distance themselves from professionally managed portfolios, and proudly claim that their strategy has nothing average, and is instead fundamentally personal.

For this reason, we decided to adopt a qualitative approach with in-depth interviews of individual investors. Our sample is made of experienced investors, who have a sustained investing practice and have found their "style" after a learning period.

In contrast to the vague but still prescriptive theories of Finance, Behavioral science aims at starting the analysis from what investors really do, and understand the rationale behind it. The notion of biases, used and abused by practitioners, is a central concept in Behavioral science. A return to its core definition might help sort between what are real systematic deviations from optimal decision-making, and what are useful heuristics that bring value-added - financial or otherwise - to the decisionmakers.

In this paper, we want to shed light on experienced individual investors' real investment practices, and question the idea of absolute rationality in contrast with ecological rationality driven by individual's motivations and capabilities.

\section{Financial theories and empirical literature}

Standard finance provides concepts, theories, and methodologies for creating investment portfolios meeting investor's return and risk preferences. The four foundation blocks are rational investors maximizing their financial utility, efficient markets, modern portfolio theory and the interdependence of risk and return (Statman, 2008).

Modern portfolio theory (MPT), is a mathematical framework for assembling a portfolio of assets such that the expected return is maximized for a given level of risk (Fabozzi et al., 2002). Since the pioneering work of Markowitz (Markowitz, 1952), diversification has been a fundamental concept in asset management and assetpricing theories. A direct implication of Efficient Market Hypothesis (EMH) is that it is impossible to "beat the market" consistently. This is the theoretical ground behind passive index investing or buy-and-hold investing.

There is an ongoing debate upon the validity of finance theories, and the credibility of sophisticated mathematical models has been further questioned following the 
financial crisis of 2007-2008. Researchers have disputed the efficient market hypothesis both theoretically and empirically.

While traditional finance assumes that an investor is a rational person capable and willing to process all information without biases, and that the only goal of investing is to maximize financial utility, Behavioral finance studies how emotional, cognitive, and psychological factors influence investment decisions. Behavioral finance helps to explain the difference between expectations of rational investor behavior and actual behavior.

Prospect theory (Kahneman \& Tversky, 1979), remains the most successful alternative to the Expected Utility Theory (Illiashenko, 2017). It is a behavioral model showing how people consider utility relative to a reference point and that utility of the same loss and gain is asymmetric. Among other, this explains the disposition bias - investors having trouble selling losing assets.

Shefrin and Statman (2002) introduced the Behavioral portfolio theory (BPT), a goalsbased theory, where investors divide their money into mental accounts relating to investing goals like financing retirement, university education or just becoming wealthy.

The existing empirical research on individual investors end up to some common findings. Individual investors as a group underperform the markets. Barber and Odean (Barber \& Odean, 2000) illustrate that the average passively investing US household earns a $1.5 \%$ lower return than the market return, whereas most active investors involved in trading underperform by $6.5 \%$ annually.

Findings of empirical research strongly supports the benefits of diversification. Goetzmann and Kumar (2008) found a 2.4\% yearly return difference between the least and the best diversified investors in the same data.

Empirical research does not support investor interest in stock-picking. (Odean, 1999) found that the stocks investors buy subsequently underperform the stocks they sell. Findings of (Grinblatt \& Keloharju, 2000) are similar: individual investors are net buyers of stocks with weak future performance.

As a group, individual investors have been found to exhibit poor market timing ability (Kamesaka et al., 2003). Barber et al. (2000) estimated investor timing to reduce the performance of individual investors by approximately 0.29 percent annually

Overconfidence is suggested to explain the excess number of transactions and part of stock selections (Barber \& Odean, 2000); (Gervais \& Odean, 2001). The poor timing of transactions has been explained by disposition bias (Shefrin \& Statman, 1985).

Barber and Odean argue that attention greatly influences individual investor purchase decisions (Barber \& Odean, 2008). Rather than searching systematically, many investors may consider only stocks that first catch their attention. 
Results of existing empirical research raise questions: what are true reasons behind investor behavior? Do they apply also to seasoned investors?

\section{Behavioral point of view}

By adopting a behavioral science point of view, we can distinguish two classes of tools for decision-making: optimization, and heuristics (Mousavi \& Gigerenzer, 2014).

Optimization can be defined as computing the utility-maximizing solution to a problem using deterministic mathematical methods, given a set of variables and constraints. It is, usually, what people have in mind when talking about a rational solution.

Heuristics are "simple strategies that ignore part of the available information" (Hafenbradl et al., 2016). Acknowledging that availability of both information and decision-maker computing power are limited, bounded (Simon, 1955), they aim at exploiting relevant characteristic of the environment in a decision process that is fast, and frugal in terms of resources. Their outcome can be as good - or even better - than optimization methods (Gigerenzer \& Todd, 1999) if they are applied in the right context and correctly calibrated, which is what the authors call ecological rationality. However, it is also known that they have the potential to lead to systematic errors, called biases (Tversky \& Kahneman, 1974) when applied out of their domain of relevance.

Modern mathematically grounded education, and spreadsheet-worshiping civilization, naturally pushes us to consider optimization as the gold standard, and heuristics as a lesser evil, to use in last resort but not without a touch of shame. Researchers belonging to the "fast-and-frugal heuristics" stream point this as a deep misconception (Mousavi \& Gigerenzer, 2017). Indeed, optimization requires parameters, the set of possible events and their probabilities, to be known, and computable. They require the decision-maker to be in a "world of risk" (Knight, 1921). If, instead, the decision-maker is in a world of uncertainty, where the set of possible events - not to mention their probabilities and possible consequences - are unknown or impossible to compute, optimization is by definition impossible, and the correct approach is to embrace heuristic decision-making and start working at matching the right heuristic to the problem at hand.

Most of our decisions, small and big, happen in a world of uncertainty. The usual mistake is to artificially reduce uncertainty to risk, in order to pretend optimization is possible, and save the face the "rationality" to justify a decision. This myopic approach leads to unpleasant surprises when uncertainty comes to reclaim its territory - crisis.

It is interesting to note that practitioners in our study tend to over-use the term "bias" to designate any decision that is not backed by data and quantitative analysis. They also reject the idea that they use heuristics, and maintain the idea that the rational 
behavior - even if they do not pretend to always adopt it - is out there. However, of all the decision-making contexts that human can face, the world of investing is arguably one of the most uncertain. Variables are infinite, complexity is maximal, and many of the most reputable theories are either contradictory - down to the very debate of market efficiency - or do not provide actionable prescriptions on what to do in practice.

The interest of the behavioral approach for our research is that there seems to be a tacit but widespread schizophrenia in investors, who recognize the uncertain nature of the market, but want at the same time to maintain an image of "rationality", that, in their mind, means a deterministic mathematical approach. A behavioral approach can help recognize what are the biases at play but also what are the perfectly ecologically rational heuristics that investors use, and maybe reconcile their self-image of rational beings with their methods.

\section{Methodology}

The research goal calls for explorative research design using qualitative semistructured interviews. The design allows gaining of a holistic overview and understanding investing in a person's context. (Miles et al., 2020) (Yin, 2011)

The primary data source was 32 investor interviews: Switzerland (14), USA (10) and Finland (11). Comparisons between countries was not the purpose of the study, but three countries with self-directed investing culture were selected to avoid single country sampling bias. A connection to a larger research project made access to this data set possible.

The main criteria for an investor to be included in the sample was a minimum investing experience of 5 years, self-directed sustainable investing style and minimum portfolio size of 50000 euros. Multiple years of investing experience enables a longitudinal research perspective. The portfolio size requirement was added with the expectation of larger variation of investing styles. Investors using only automatic investing or discretionary accounts were excluded.

Expert interviews (8) were a secondary information source. By experts is meant professional asset managers, relationship managers, portfolio managers and advisors. Experts provided a new perspective helping to interpret and confirm the findings while limiting potential interview method biases (Eisenhardt \& Graebner, 2007).

The main interview phase took place between February - December 2019. Of the 32 interviews, 20 were face-to-face interviews and 12 were telephone interviews. Interviews were recorded with those interviewees permitting recording of this highly confidential information. The interview agenda was organized into the following interview themes:

- Investor's investing history 
- Current investing style

- Perceived investing performance

- Characteristics of current portfolio(s)

- Portfolio management style

- Analyses and decision-making

- Behavioral issues: self-assessment and researcher inquiry

- Reasons for not following common investing guidelines

Use of themes as a flexible interview guide follows the recommendation of qualitative research using protocols by (Yin, 2011).

The analysis method used is an adaptation of elements from Grounded Theory, Gioia Method (Gioia et al., 2013) and method introduced by Miles \& Huberman (Miles \& Huberman, 1994). Characteristics of the method include evolution of interview protocol, partially framework free initial coding and continuous analysis. Predefined hierarchic coding was used for established concepts related to portfolio management and behavioral finance.

\section{Observed investing patterns}

\section{Investor experience cycle, learning process and sustainable investing phase}

The first questions of the interviews explored the investors' reasons to start investing and their life story of investing. Narratives of initial stages in the self-managed investing career often included actions of trial-and-error or experimental nature; exsample people who had ceased investing were asked for the reasons behind their decision to quit. Figure 1 below depicts identified paths from a decision to start investing onwards.

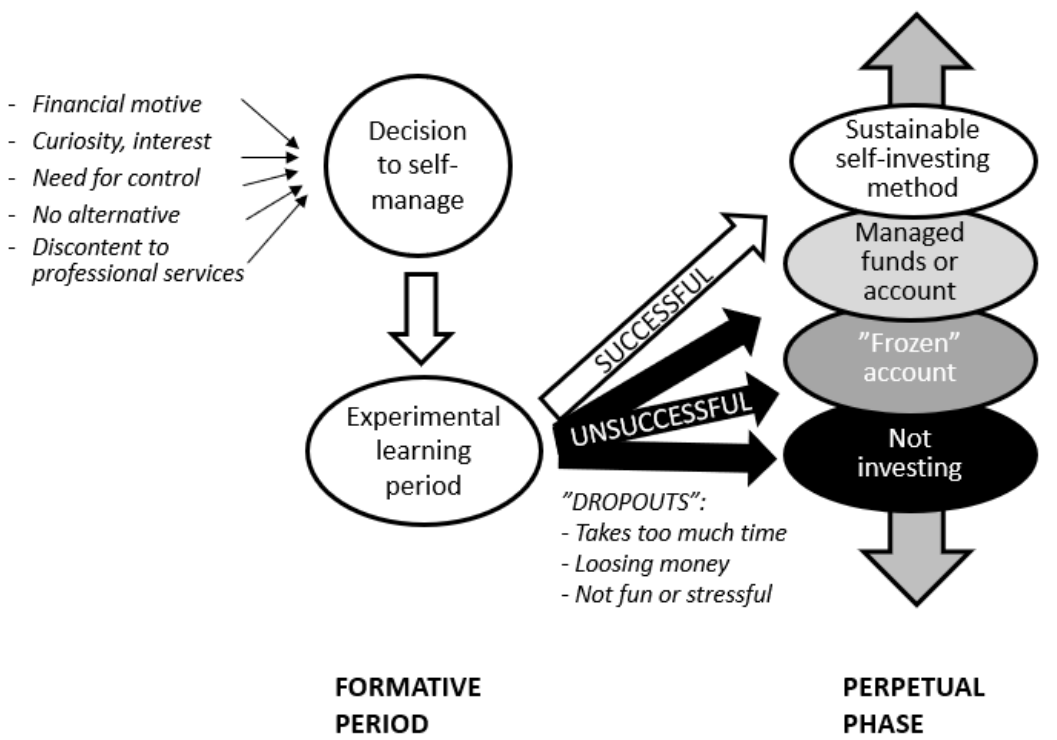


Figure 1. Investor experience cycle: formative period and perpetual phase. The investors in the interview sample started investing from various reasons. The initial learning period is named Formative Period. In the Perpetual Phase, they have found a sustainable way of investing. Frozen account means, that with disappointing results, accounts were left aside with no systematic management.

Common narratives included realizing one's own limits, need to understand instead of guessing or following others, becoming more passive, taking a longer investing horizon, becoming more selective in which securities to invest and not trusting secondhand information.

\section{Motivation for investing}

All investors had some form of financial objective for investing, if not more than "saving for the future". An unexpected finding was the importance of mental utility for self-directed investing. Generally distrusting commercial financial services, most investors wanted to feel being in better control of their financial assets. Many investors found some of the investing tasks enjoyable, like the search of next unicorns or the ideation future overperforming industrial sectors. Further mental satisfaction was derived from discussions with peers (belonging), supporting important themes (examples: renewable energy, sustainability, medical innovations) and selfactualization "[...] this blond can make millions".

\section{Investing practices confirming with traditional finance expectations for rational investing}

In the sample there was no criticism of the benefits of buy \& hold investing strategy. In some literature (Reilly \& Brown, 2011), buy \& hold is synonymous with passive index investing, but in investor cases it meant keeping selected securities in the portfolio, unless there is an compelling reason to switch securities. In spite of all investors in the sample having a passive portfolio allocation, the more active investors kept also an allocation for more opportunistic investments. Opportunistic investing did not mean trading for short-term, but looking for new promising investing occasions.

No investor posited that market timing is possible. Still, when increasing investments, investors admitted looking for advantageous moments to acquire single securities.

A discovered contemplating and sense-making decision-making pattern was discovered, hereafter called Decision Incubation. Instead of reacting to single market events, investors spent considerable calendar time in collecting information and analyzing it.

Investing practices considered irrational from financial utility and portfolio theory points of view

From portfolio theory point of view, no investor had an efficient portfolio - and this was not a goal for investors. However, benefits of diversification were well 
understood as " the only free lunch in investing", but not fully implemented due to practical reasons or perceived lack of importance.

Investors showed a strong priority on their individual investment positions and less interest for their portfolio as a whole. Only three investors started describing their investing portfolio from asset allocation point of view. Only one investor mentioned an explicit rebalancing practice.

According to efficient market hypothesis, it is impossible to overperform the market in long term, but four investors in the sample claimed overperforming the market index. One of these investors defended his overperformance claim by disclosing a spreadsheet showing a decade of overperformance

An unforeseen result was the investors common unawareness or ignorance of their portfolio performance. On the other side, the investors who did not know their portfolio performance, were often well aware of the under/overperformance of single assets in the portfolio. When asked does their portfolio reach or exceed market indices, they typically replied with disbelief or uncertainty - a representative quote: "I don't know accurately, but I guess not".

Literature review revealed the normative top-down investing process focusing on optimizing the portfolio as a whole; the process starts from investor needs and ends to investment selection for the planned target portfolio. Instead, a large majority of investors followed a bottom-up investing process starting from identification of winning investments. Depending on investor, portfolios were reactively and intuitively balanced later. Only one investor was able to show a written investing plan.

\section{Observed Behavioral and Decision-making Patterns}

It is to be noted that "bias" or "biased" are understood by investing practitioners as broader concepts than in behavioral science literature. By practitioners, the use of the term varies from biased decision-making to a synonym for bad decision-making.

\section{Findings conforming with existing literature}

Investor and expert comments demonstrated the existence of disposition bias by describing their emotions related to asset transactions. Affect bias appeared in the form of strong investor interest to talk about their favorite securities. A clear pattern found was investors' fear of their own emotions affecting rational decision-making. Other mentioned biases expressed by investors themselves were recency bias prioritization of recent information and home bias - favoring domestic securities.

\section{Findings not conforming with existing literature}

Overconfidence often referred in literature could not be identified. Instead, investors frequently described themselves feeling not confident. Illusion of control suggested in empirical literature due to online platforms could not be identified - possible reasons including preparation of decisions outside platforms or gained experience. 
Herding could not be identified. The following of the often successful "trend is your friend" heuristics - favoring assets growing in value or momentum investing strategy cannot be considered as herding.

\section{New patterns observed}

A new behavioral bias tangibility bias is suggested. This strong pattern means that an investor is ready to accept higher risk or lower financial utility if he better understands his portfolio positions. An example is avoiding well-diversified highquality funds as funds feel like a black box. It can also exhibit as preference of underdiversified portfolios where security positions are well known. Whether tangibility bias is a bias or rational reasoning depends on how it systematically affects the quality of decision-making. The matter deserves confirmation through research with experimental design.

Unlike overconfidence, the pattern of underconfidence was encountered. The finding was confirmed by two experts. Underconfidence is a bias, if it systematically leads to unoptimal portfolios like large chronic allocations of uninvested assets. After anecdotes, amount of underconfidence can vary along time.

A seemingly experience originated decision-making pattern was self-mitigation of behavioral biases and emotions. This strong pattern made investors take proactive or reactive actions to mitigate effects of what they perceive as their biases. Proactive measures included accumulating knowledge and selecting lower risk portfolios. Reactive measures mentioned included mentally mitigating losses by putting performance in a longer perspective, applying ostrich method (Galai \& Sade, 2006) or talking with advisors and peers. Several investors considered proactive measures the only effective way of mitigating compromised decision-making. Learning to mitigate biases and emotional decision-making implies that the effect of biased decisionmaking may vary along time.

Some investors had strong convictions developed from their own good/bad experiences. Based on our subjective assessment, convictions can conform with mainstream financial paradigm, like "You cannot beat the market" or be apparently biased (example: "Buy \& hold is dead. You need to take your profits"). We assume that convictions may harm investing performance if based on insufficient or misinterpreted evidence. A financial expert interviewed divided convictions to obsessive or convertible. Convertible convictions could be removed or changed, but obsessive ones are self-defeating.

Analyzing investor decision-making patterns revealed deviations from commonly accepted investing tenets and empirically proven guidelines. Instead of calling this type of behavior "irrational" we suggest a concept contextual investing rationality. Departures from the norm were seldom based on only ignorance but also on deductive inference and non-financial motives. Examples: having a "biased" portfolio with a sectoral overweight by an investor with special sectoral knowledge, preference 
for a high-risk portfolio "[...] as my investing horizon is 30 years" or just enjoying to invest in companies "[...] with a good purpose".

\section{Motivation and Experience, the Importance of Personal Aspects}

Two characteristics of our sample are key to the behavioral analysis: the diversity of investment motivations, and the self-selection through a dropout process.

While investing is intuitively associated with the wish to make profit, participants in the interviews mention many motivating factors beyond financial. These factors, such as curiosity, enjoyment, and need for control, suggest that the motivation is not only extrinsic - the expectation of a financial reward - but also intrinsic to the investment activity. Therefore, the outcome of their experience cannot be reduced to the benchmark of their portfolio performance against the market or professional portfolios.

More importantly, deviations from investment theory - such as heightened interest in stock-picking - that might be detrimental to financial performance, needs to be analyzed on other dimensions too, as an overweight bet on a specific company can bring an investor significant satisfaction in terms of belonging feeling and selfactualization. These investors' utility function is a complex mix of financial and higher-level factors of the Maslow pyramid, and what is seen as biases by the portfolio-balancing solver is in fact ecologically rational when the model is calibrated to maximize the true and complex objectives of decision-maker.

Despite their paramount importance, these investment objectives remain elusive in the evaluation of investor experience. Our interviewees are able to identify various motivators when prompted about it, but the assessment of their performance is reduced to a measure of return on investment, that they often do not know. We suggest that a reason for that might be the absence of place for intrinsic motivation factors performance evaluation in investment frameworks, that are usually designed for professional investors and therefore focus on financial performance only.

The second important aspect of our sample is its self-selection aspect. Our participants are sustained investors who decided to carry on with individual investment after what we called experimental learning period depicted in Figure 1.

It is interesting to ask whether this process is purely passive - people who are not made for individual investing stop and those who are remain - or if it is possible for an apprentice to adapt, improve, and actively become a better individual investor.

(Klein, 2001) suggests that gaining expertise in a domain requires, beyond just accumulating experience, to understand the big picture. It might sound like an ambitious feat in an environment such as financial markets where feedback is not straightforward, delayed, and impact of decisions can easily be lost in the noise of general market performance. 
Our interviewees described changes in their investing behavior that draw the picture of a calibration of their heuristics: common narratives included realizing one's own limits - a transition from an ideal of maximization to satisficing in the sense of (Simon, 1956) - need to understand instead of guessing or following others, becoming more passive, taking a longer investing horizon, becoming more selective in which securities to invest and not trusting second hand information.

The fact that these investors reached a steady state makes their set of heuristics worth studying as it suggests that they developed skills towards an expertise that allows them to get satisfaction out of the investment activity. Their experience could be used to develop a framework focused on individual investors, considering both financial and personal aspects.

\section{Conclusion}

Studies of investors and their practices are too often based on faceless and pooled quantitative trading data, or worse, skewed by samples overwhelmed by beginning experimenters or traders who do not represent investors. In this paper, we take advantage of a unique dataset of in-depth interviews with experienced investors, who went through a learning period, did not drop out, and found their personal sustainable investing practice.

We notice some convergence to practices that are in line with financial tenets, but we also identify ongoing behaviors that go against theoretical prescriptions: position focused stock-picking, sectorial overweight and underdiversified portfolios seldom rebalanced.

While these practices are often turned down under the overused label of biases, we argue that - at least to some extent - they might correspond to purposes and motivations of personal investment that go beyond just financial returns - a benchmark that is not even closely monitored by our investor sample. In this regard, what looks like irrational quirks are in fact effective heuristics that have an ecological rationality value. A "too" concentrated but tangible portfolio may better meet an investor's financial and mental needs.

To build on this exploration and to go further, there are two tracks to go. The first track involves building an experimental research design that allows for a formal testing of features identified in the interviews, such as the suggested tangibility and underconfidence biases. The second track moves on exploring: how online platforms and continuous information overflow affect investor behavior; how the increasing share of exchange traded funds in portfolios changes the investor mindset.

\section{References}

[1] Barber, B. M., \& Odean, T. (2000). Trading is hazardous to your wealth: The common stock investment performance of individual investors. The Journal of Finance, 55(2), 773-806. 
[2] Barber, B. M., \& Odean, T. (2008). All that glitters: The effect of attention and news on the buying behavior of individual and institutional investors. The Review of Financial Studies, 21(2), 785-818.

[3] Eisenhardt, K. M., \& Graebner, M. E. (2007). Theory building from cases: Opportunities and challenges. Academy of Management Journal, 50(1), 2532.

[4] Fabozzi, F. J., Gupta, F., \& Markowitz, H. M. (2002). The legacy of modern portfolio theory. The Journal of Investing, 11(3), 7-22.

[5] Galai, D., \& Sade, O. (2006). The "ostrich effect" and the relationship between the liquidity and the yields of financial assets. The Journal of Business, 79(5), 2741-2759.

[6] Gervais, S., \& Odean, T. (2001). Learning to be overconfident. The Review of Financial Studies, 14(1), 1-27.

[7] Gigerenzer, G., \& Todd, P. M. (1999). Fast and frugal heuristics: The adaptive toolbox. In Simple heuristics that make us smart (pp. 3-34). Oxford University Press.

[8] Gioia, D. A., Corley, K. G., \& Hamilton, A. L. (2013). Seeking qualitative rigor in inductive research: Notes on the Gioia methodology. Organizational Research Methods, 16(1), 15-31.

[9] Goetzmann, W. N., \& Kumar, A. (2008). Equity portfolio diversification. Review of Finance, 12(3), 433-463.

[10] Grinblatt, M., \& Keloharju, M. (2000). The investment behavior and performance of various investor types: A study of Finland's unique data set. Journal of Financial Economics, 55(1), 43-67.

[11] Hafenbradl, S., Waeger, D., Marewski, J. N., \& Gigerenzer, G. (2016). Applied decision making with fast-and-frugal heuristics. Journal of Applied Research in Memory and Cognition, 5(2), 215-231.

[12] Illiashenko, P. (2017). Behavioral finance: History and foundations. Visnyk of the National Bank of Ukraine, 239, 28-54.

[13] Kahneman, D., \& Tversky, A. (1979). Prospect Theory of Decisions Under Risk. Econometrica, 47(2), 1156-1167.

[14] Kamesaka, A., Nofsinger, J. R., \& Kawakita, H. (2003). Investment patterns and performance of investor groups in Japan. Pacific-Basin Finance Journal, 11(1), 1-22.

[15] Klein, G. (2001). Sources if Power: How People make Decisions. London: MIT Press.

[16] Knight, F. H. (1921). Risk, uncertainty and profit (Vol. 31). Houghton Mifflin.

[17] Markowitz, H. (1952). Portfolio Selection, Journal of Finance. Markowitz HM-1952. - №, 77-91.

[18] Miles, M. B., \& Huberman, A. M. (1994). Qualitative data analysis: An expanded sourcebook. sage.

[19] Miles, M. B., Huberman, M. A., \& Saldana, J. (2020). Qualitative data analysis: A methods sourcebook. SAGE. 
[20] Mousavi, S., \& Gigerenzer, G. (2014). Risk, uncertainty, and heuristics. Journal of Business Research, 67(8), 1671-1678.

[21] Mousavi, S., \& Gigerenzer, G. (2017). Heuristics are tools for uncertainty. Homo Oeconomicus, 34(4), 361-379.

[22] Odean, T. (1999). Do investors trade too much? American Economic Review, 89(5), 1279-1298.

[23] Reilly, F. K., \& Brown, K. C. (2011). Investment Analysis and Portfolio Management. Cengage Learning.

[24] Shefrin, H., \& Statman, M. (1985). The disposition to sell winners too early and ride losers too long: Theory and evidence. The Journal of Finance, 40(3), 777-790.

[25] Simon, H. A. (1955). A behavioral model of rational choice. The Quarterly Journal of Economics, 69(1), 99-118.

[26] Simon, H. A. (1956). Rational choice and the structure of the environment. Psychological Review, 63(2), 129.

[27] Statman, M. (2008). What is behavioral finance? Handbook of Finance, 2.

[28] Tversky, A., \& Kahneman, D. (1974). Judgment under uncertainty: Heuristics and biases. Science, 185(4157), 1124-1131.

[29] Yin, R. K. (2011). Qualitative research from start to finish. New York, NY. Guilford Publications, Inc. 